\title{
Palomar Testbed Interferometer: update
}

Benjamin F. Lane, M. Mark Colavita, Andrew F. Boden, Peter R. Lawson

Benjamin F. Lane, M. Mark Colavita, Andrew F. Boden, Peter R. Lawson, "Palomar Testbed Interferometer: update," Proc. SPIE 4006, Interferometry in Optical Astronomy, (5 July 2000); doi: 10.1117/12.390239 


\title{
Palomar Testbed Interferometer - Update
}

\author{
B.F. Lane ${ }^{a}$, M.M. Colavita ${ }^{b}$, A.F. Boden ${ }^{b}$, P.R. Lawson $^{b}$ (for the PTI Collaboration) \\ ${ }^{a}$ Department of Geological \& Planetary Sciences, California Institute of Technology \\ 150-21, Pasadena, CA. 91125, USA. \\ ${ }^{b}$ Jet Propulsion Laboratory, California Institute of Technology, \\ 4800 Oak Grove Dr., Pasadena, CA., 91109, USA.
}

\begin{abstract}
The Palomar Testbed Interferometer (PTI) is a long-baseline near-infrared interferometer operating at Palomar Observatory, CA. The interferometer has a maximum baseline of $110 \mathrm{~m}, 40-\mathrm{cm}$ collecting apertures, and active fringe tracking. It also incorporates a dual-star architecture to enable cophasing and narrow-angle astrometry.

We will discuss recent system improvements and engineering results. These include upgrades to allow for longer coherent integration times, $\mathrm{H}$ band operation, and cophasing using delay line feedforward. Recent engineering tests of astrometry in dual-star mode have shown a night-to-night repeatability of $100 \mu$ as on a bright test target. Several new observation planning tools have been developed, and data reduction tools have been automated to allow fully pipelined nightly reductions and archiving.
\end{abstract}

Keywords: Interferometry, Instrumentation, Astrometry, Planet-detection

\section{INTRODUCTION}

The Palomar Testbed Interferometer (PTI) is a long-baseline infrared interferometer installed at Palomar Observatory, California. It operates in the $\mathrm{H}(1.6 \mu \mathrm{m})$ and $\mathrm{K}(2.2 \mu \mathrm{m})$ bands, and with a maximum baseline of $110 \mathrm{~m}$ achieves an angular resolution of $\sim 3$ milli-arcseconds. It was developed by the Jet Propulsion Laboratory, California Institute of Technology for NASA, as a testbed for interferometric techniques applicable to the Keck Interferometer as well as other missions such as the Space Interferometry Mission, SIM. PTI has been used in the development of highsensitivity direct-detection interferometry in the infrared with array detectors, phase-referencing, and narrow-angle astrometry. PTI also serves as a testbed for interferometric planning, operational techniques, and data processing and management tools applicable to both ground and space-based interferometers.

Major development of PTI began in November 1992 with the commencement of funding from NASA under its TOPS program. The interferometer was installed at Palomar Observatory during the spring of 1995, and first fringes were obtained in July 1995. Initial astrometric measurements were made starting in 1997, achieving 100 $\mu$ as accuracy in 1999. Phase referencing results using long integration times were also first obtained in 1999.

\section{THE INSTRUMENT}

The Palomar Testbed Interferometer ${ }^{1}$ was designed with an unusual "dual-star" configuration in which the image plane of the apertures can be split (usually by a 50-50 beam-splitter) such that light can be directed down two different beam paths to two separate beam combiners. Thus it is in effect two independent two-aperture interferometers that share the same apertures. In general one star (called the "primary") is observed on-axis, while the "secondary" star can be anywhere within an annulus with inner radius $\sim 8$ arc-seconds (closer than that and the tip-tilt sensor confuses the primary and secondary stars) and an outer radius of 1 arc minute.

After tip-tilt correction by a fast steering mirror, the starlight from each aperture passes through optical delay lines $^{2}$ to correct for geometric and atmospheric optical path-length differences. PTI was designed such that both the primary and secondary starlight beams pass through a common long delay line ("LDL", capable of up to $\pm 38.3 \mathrm{~m}$ of optical delay), after which only the primary beam passes through a short delay line ("SDL", $\geq 3 \mathrm{~cm}$ of optical delay, corresponding to $\geq \pm 1$ arc-minute on the sky). In effect, the primary fringe tracker sees a "primary" delay line with an optical delay given by $\delta_{p}=\delta_{L D L}+\delta_{S D L}$, while the secondary fringe tracker sees a "secondary" delay line with

Send correspondence to B.F.L. : E-mail: ben@gps.caltech.edu 
delay $\delta_{s}=\delta_{L D L}$. The delay line controller orthogonalizes the commands sent to the physical delay lines such that the fringe trackers can request optical pathlength changes to the primary and secondary delay lines independently. Also, delay modulation (see below) can be applied to either or both of the delay lines.

Optical pathlengths are monitored by several laser metrology gauges, including independent LDL and SDL monitors, as well as a "constant-term" (CT) metrology system, which measures the total difference in optical path between the primary and secondary beams throughout the entire optical system out to the apertures. The SDL can use either its local metrology system or the CT to determine position. The latter case provides a way to compensate for piston vibrations of the optics in the starlight path and is used in phase referencing.

The tip-tilt corrected and delay compensated starlight beams from each aperture are combined at a 50-50 beamsplitter. The output of the beam-splitter is two combined beams, one of which is focussed directly onto a single pixel of a NICMOS-III detector. The other beam is first spatially filtered by passage through a single-mode fiber and then dispersed with a prism before being focussed onto 5-10 (depending on the chosen spectral resolution, typically 65 $\mathrm{nm} / \mathrm{pixel}$ ) pixels on the same detector, and is used as a spectrometer. The fringe signal is measured by modulating the delay by one wavelength in a sawtooth pattern and synchronously reading out the detector. During each sample the detector is first reset, a bias level is read, and then 4 reads are done, one after each quarter-wavelength of modulation. These four reads are used to solve for fringe visibility and phase. Fringe tracking is done by feeding back the unwrapped phase to the primary delay line via an integrating servo (Figure 2).

PTI is able to operate in three fundamental modes: the simplest is the case when only one fringe tracker operates, tracking and measuring the visibility of a single star. In this mode the 50-50 beam-splitters in the focal planes of the apertures are usually removed, increasing the photon throughput of the instrument. The second observing mode is used for astrometric measurements of similar-magnitude visual binary systems: in this mode both the primary and secondary fringe trackers operate independently with short sample times, and the primary and secondary delay lines are effectively independent. The third available mode is phase-referenced mode, in which the primary fringe tracker tracks a bright $\left(m_{K}<4.5\right)$ star with short sample times, while correcting the measured phase error for both the primary and secondary stars. In this mode the secondary fringe tracker can operate with integration times of 100 ms or longer, achieving a limiting magnitude of at least 6th mag. A detailed description of PTI is also available in reference 1.

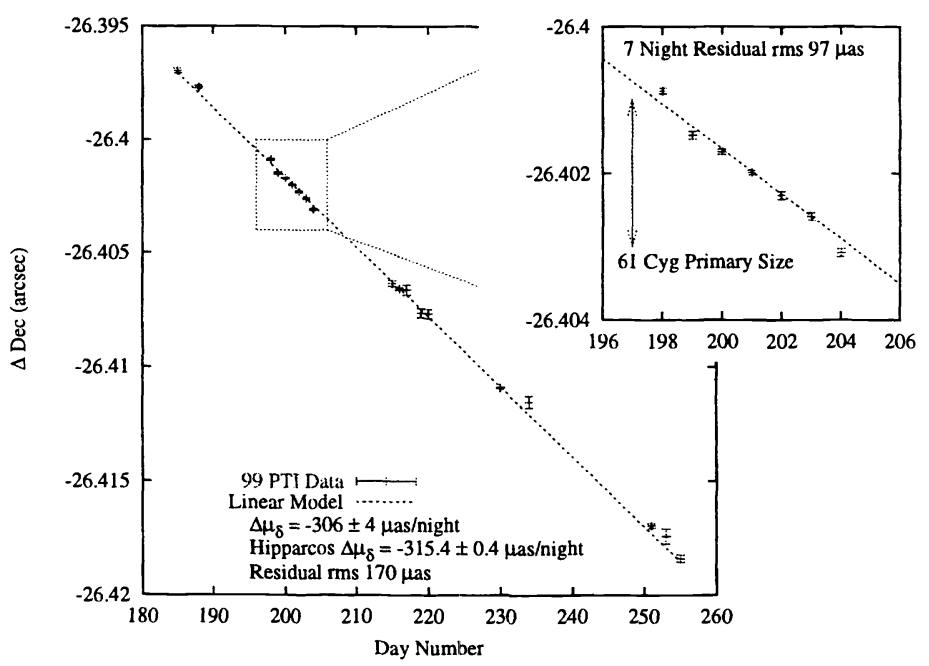

Figure 1. The differential Declination between the components of $61 \mathrm{Cyg}$, as measured by PTI in 1999 . 


\section{ENGINEERING RESULTS}

\subsection{Astrometry}

The development of technology for detecting extra-solar planets through astrometry is one of the motivations for building PTI. While high-accuracy global astrometry remains the purview of space missions, the astrometric detection of extrasolar planets is primarily a narrow-angle problem, as the measurement can be made with respect to angularly nearby reference stars. In this case, the atmospheric limits to astrometric accuracy achievable with a long- baseline interferometer are several orders of magnitude below the wide-angle atmospheric limit. For interferometer baselines of $\sim 100 \mathrm{~m}$, and star separations of 15-20 arc-sec, accuracies of tens of microarcseconds in an hour measurement are possible. $^{3}$

During the summer of 1999 we observed the bright visual binary 61 Cygni (HD 201091/201092, K5V+K7V) to determine the astrometric stability of PTI. After numerous hardware improvements implemented as a result of our 1998 experience, 1999 data on 61 Cyg exhibits astrometric precision of 100 microarcseconds $\left(10^{-6}\right.$ arcseconds, $\mu$ as $)$ over a one week time-scale, and $170 \mu$ as rms precision over a 70 day time-scale. Further, the 61 Cyg component differential proper motion measured by PTI in 1999 is in good agreement with the Hipparcos determination of system motion (Figure 1).

PTI astrometric measurements of 61 Cyg will continue in the 2000 observing season with the objective of establishing a three-year baseline for proper motion and relative parallax determination, and astrometric measurements using the quasi-orthogonal $\mathrm{N}-\mathrm{W}$ baseline configuration to demonstrate similar $2 \mathrm{~d}$ performance.

\subsection{Phase Referencing}

By sythetically increasing the apparent atmospheric coherence time, phase-referencing promises a dramatic increase in the sensitivity of a stellar interferometer. This increase in sensitivity is necessary before large-scale planet searches can be initiated.

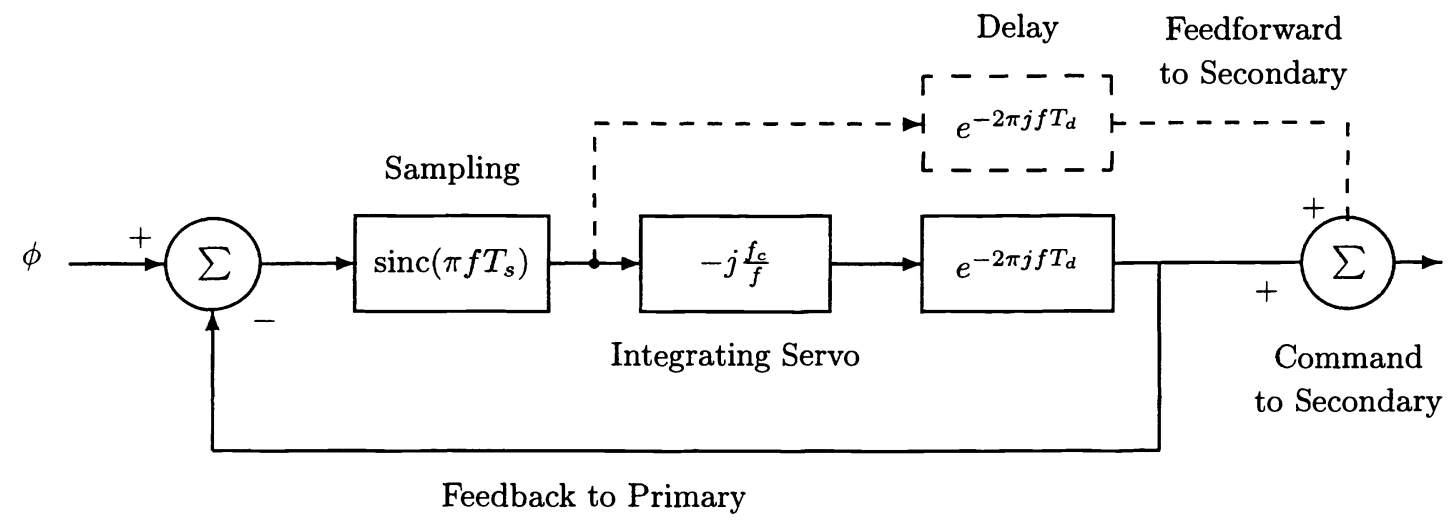

Figure 2. The control loop as implemented at PTI. The primary fringe tracker operates a feedback loop, while the secondary is phase-referenced either by sending the same integrated error signal to both primary and secondary, or by feeding forward the full error signal to secondary.

There are two ways to implement phase-referencing at PTI: the first is to simply apply the same servo correction to both the primary and secondary delay lines. Unfortunately, for stability reasons the feedback loop of the primary fringe tracker can only apply a fraction of the instantaneous phase error per cycle (i.e. the servo gain $<1$ ), limiting the performance of the fringe tracker. However, since the secondary delay line is not part of a feedback loop, no such stability criteria apply to it, and the full phase error can be applied. In this case the measured (by the primary FT) phase error is fed forward without being integrated or scaled by the gain factor to the secondary delay line. For a feedforward case such as this, the performance of the system is determined by the integration time, and the time 
delay between the phase measurement and the application of the correction. As long as the time delay is reasonably short, the feedforward case should result in reduced servo error compared to the feedback case.

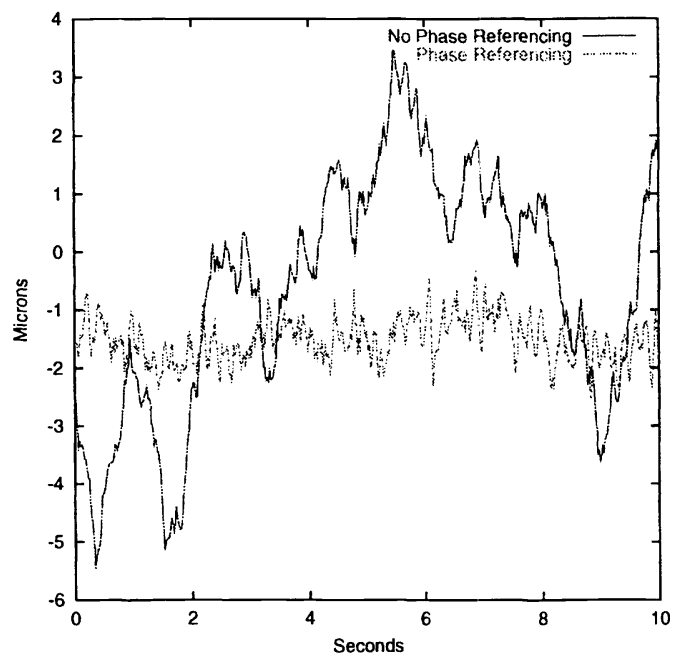

Figure 3. Unwrapped fringe position seen by the secondary fringe tracker with and without phase referencing. The two data sections were taken on $4 \mathrm{Aug}, 1999$, within 200 seconds of each other. The target star was HD 177724, $\left(m_{K}=2.99, \mathrm{A0V}\right)$. The fringe tracker was operating with $20 \mathrm{~ms}$ sample times, open loop, i.e. measuring but not correcting the phase.

Initial results from phase-referencing experiments at PTI are encouraging, and show that it is possible to increase coherent integration for the secondary times by at least a factor of 10 . Using long integration times, we have demonstrated fringe tracking on an $m_{K}=5.6$ target, an improvement over previous performance by a magnitude. However, the system is not yet meeting theoretical performance expectations. The most likely cause is imperfect correction of internal vibrations. Nonetheless, phase-referenced measurements of source visibilities can be calibrated to the $2-10 \%$ level, depending on source brightness and observing conditions. Future experiments will be conducted to determine the ultimate improvement possible, as well as the effect of anisoplanatism.

\section{SYSTEM IMPROVEMENTS}

In addition to the work on astrometry and phase referencing, several improvements were made to the instrument during 1999. These resulted in increased sensitivity, higher spatial resolution and broader spectral coverage.

\subsection{Variable Integration Time}

The fringe tracker at PTI initially operated with a fixed sample time of 10 milliseconds, tailored to the expected atmospheric coherence time. However, subsequent observational experience indicated that approximately $50 \%$ of nights during the summer observing season had a significantly calmer atmosphere, allowing longer coherent integration times. Thus it was decided to take advantage of this by modifying the fringe tracker and array control software to allow the user to select either 10 or 20 millisecond integration times.

The change in integration time requires some recalibration of the instrument, including detector background levels and a recalibration of the pathlength dither piezoelectric actuator. The entire process takes approximately 5 minutes for an experienced observer, and is usually done no more than twice during a night. With the 20 millisecond integration time the limiting magnitude of the instrument is increased to $\geq 5$ th mag in $\mathrm{K}$. 


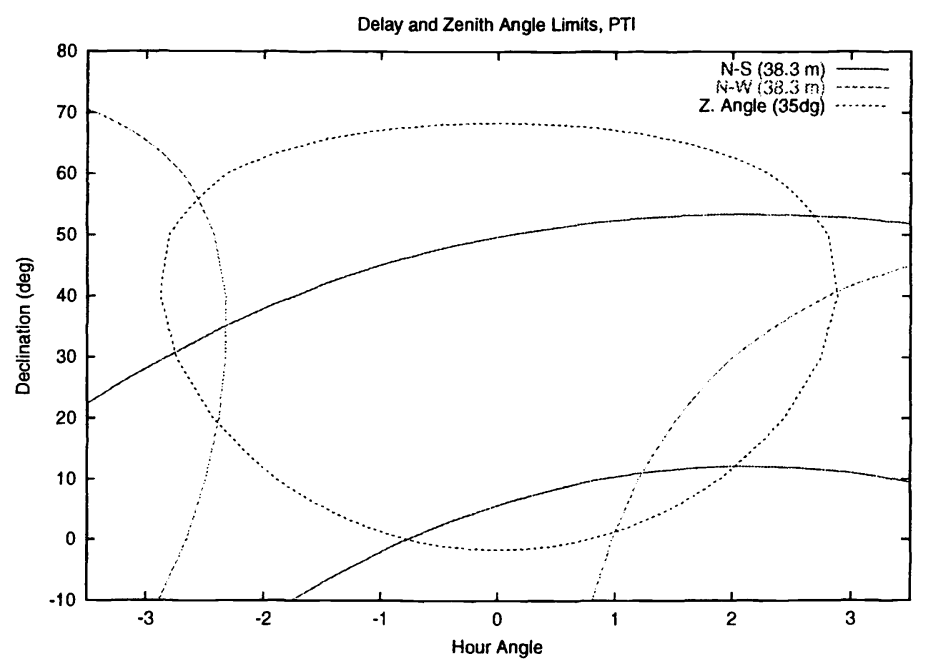

Figure 4. The sky coverage of the two PTI baselines, and the limit imposed by requiring the zenith angle to be less than 35 degrees.

\subsection{H-band Operation}

Although initially designed for operation in the $\mathrm{K}$ band $(2.2 \mu \mathrm{m})$, it has proven possible to extend the spectral range of operation of the instrument to the $\mathrm{H}$ band $(1.6 \mu \mathrm{m})$ with only slight hardware modifications. The PTI spectrometer can now be operated in 4 different modes: low-resolution $K$ provides 5 spectral channels across the $\mathrm{K}$ band $(2.0-2.4 \mu$ $\mathrm{m})$, low-resolution $H$ provides 4 spectral channels across the $\mathrm{H}$ band $(1.5-1.7 \mu \mathrm{m})$, high-resolution $K$ provides 10 channels across $\mathrm{K}$, and finally combined $H-K$ covers the $\mathrm{H}$ and $\mathrm{K}$ bands with 8 channels.

Switching spectrometer mode involves manual selection of the appropriate external prism and dewar filter, some optical re-alignment, and re-calibration of the detector and pathlength dither actuator. The process is usually done as part of the nightly set-up procedure.

Operation of PTI in the $\mathrm{H}$ band improves the spatial resolution of the instrument, allowing one to resolve e.g. nearby M-dwarf stars, as well as several of the closest Cepheid variable stars. The limiting magnitude of the instrument in the $\mathrm{H}$ band is $\sim 4.5$ mag.

\subsection{Future Developments}

In addition to the above described upgrades, several more system improvements are in various stages of planning and completion. The most notable are an additional baseline and a new detector.

\subsubsection{Additional Baseline}

PTI has a third siderostat installed west of the current North-South baseline, providing an 80-meter baseline rotated $\sim 50$ degrees with respect to the existing baseline. This baseline will substantially improve the PTI sky coverage, in particular allowing PTI to observe high-declination objects (Figure 4). This baseline will also be used to provide improved astrometric knowledge of observed sources in a Right Ascension (the current N-S baseline provides highaccuracy astrometry only in Declination). Current plans are for this second baseline to be operational in the summer of 2000 . At present there are no plans for simultaneous three-aperture beam combination, as the necessary delay line does not exist.

Switching between the North-South and the new North-West baseline involves moving two mirrors (placed on kinematic mounts for repeatability), running a script to reconfigure the necessary software, rebooting the instrument, and re-calibrating the detector and delay lines. Current plans call for switching baseline configuration only during the day. 


\subsubsection{Low-Background Dewar and HAWAII Detector}

PTI has also obtained a new low-background dewar and detector. The current warm, slitless spectrometer will be moved inside the dewar to improve the thermal background. The new detector is a Rockwell/Boeing HAWAII $1024 \times 1024 \mathrm{HgCdTe}$ array. The lower dewar background and lower read-noise detector should improve the spectrometer performance, and allow higher spectral resolution $(\mathrm{R} \sim 90)$. We hope to have the new system operational by June, 2000.

\section{SOFTWARE DEVELOPMENTS}

In addition to hardware improvements and engineering development, considerable work has been directed toward the goal of providing observation planning tools, quick-look data analysis tools, and an automated data processing pipeline.

\subsection{Observation Planning Tools}

We have observed that the inherent visibility response (the system visibility) of PTI is variable on a $\sim 30$ minute timescale, as well as being spatially variable, exhibiting $5 \%$ changes over 10 degrees on the sky. ${ }^{4}$ We attribute this mostly to the time-variable nature of atmospheric turbulence both in the upper atmosphere and within the interferometer itself, although changing instrumental vibrations as the instrument pointing changes is also thought to play a role.

In order to produce accurate visibility measurements it is thus necessary to calibrate the data by observing sources of known or accurately estimated diameter (and thus visibility) in close conjunction, both in time and sky location, with the target of interest. This requires the observer to find $1-3$ suitable calibrators for each science target. We have developed a suite of tools to facilitate this process. Given the source name in one of several standard nomenclatures the program will query the SIMBAD database for source coordinates and then automatically search the Hipparcos catalog for suitable calibrators, based on user-defined criteria such as magnitude, spectral type, size and angular distance from the science target. The user is presented with a list of candidates and associated information, including $\mathrm{V}$ and $\mathrm{K}$ magnitudes, angular distance from science target, possible binary companions (which would make the candidate unsuitable as a calibrator) and expected angular diameter. The angular diameter is estimated by several different methods, including fitting a blackbody model to archival photometry of the candidate to solve for effective temperature and apparent angular diameter. Finally the user is presented with a chart showing the times when the sources will be observable with the instrument, and well as sunset/sunrise times.

These planning tools were written using a combination of the Perl scripting language and $\mathrm{C} / \mathrm{C}++$, and provide either a command line or GUI-based interface.

\subsection{Quick-Look Data Processing}

We find that having science data available in real-time is a very useful diagnostic tool for the observer. For that reason we have developed software to quickly process the low-level data coming out of the instrument into a more manageable form. The code uses calibration data obtained as part of the nightly instrumental set-up to process, and averages the raw 10 or 20 -millisecond data into 25 second blocks. The resulting data includes fringe visibility, fringe phase jitter, source photon rates and delay line positions, and is made available in quasi-realtime to the observer. It is also made available on the instrument website to allow remote observers to access the data.

\subsection{Automated Data Processing Pipeline}

During normal operation the instrument produces relatively large $(300-800 \mathrm{Mbyte} /$ night depending on mode) amounts of data. This also includes calibration data taken throughout the night, e.g. detector dark levels and relative intensities in the two arms of the interferometer. At the end of each night of observing all this data is processed to produce $\sim 100$ Kbyte of calibrated data. This calibrated set contains visibility, source brightness and delay-line metrology information and is used in later data analysis.

We have automated the end-of-night data reduction process to simplify the observers task and provide consistent processing. An end-of-night script does the data reduction and calibration, solves for baseline and siderostat geometry, transfers the reduced data set to computers located at JPL in Pasadena for safekeeping, stores the unreduced lowlevel data into writable CD-ROM, and then sends a nightly observing report to the members of the PTI collaboration via e-mail. Finally the reduced data is made available for further analysis via posting on the World Wide Web. 


\section{CONCLUSION}

PTI has undergone several substantial improvements during the past year, including an extension of the observable spectral range and in increase in sensitivity by a factor of two. In addition considerable progress has been made toward the program goal of microarcsecond- level phase-referenced astrometry. Finally lessons have been learned regarding data calibration and processing, and these have led to the development of a highly automated and user-friendly data processing pipeline.

\section{ACKNOWLEDGMENTS}

PTI was developed by the Jet Propulsion Laboratory, California Institute of Technology, under contract with the National Aeronatics and Space Administration. Funding for the development of PTI was provided by NASA under its TOPS (Toward Other Planetary Systems) and ASEPS (Astronomical Studies of Extrasolar Planetary Systems) programs, and from the JPL Director's Discretionary Fund. Ongoing funding has been provided by NASA through its Origins Program and from the JPL Directors Research and Development Fund.

\section{REFERENCES}

1. M. Colavita et al., The Palomar Testbed Interferometer, ApJ, 510, pp. 505-521, 1999.

2. M. Colavita et al., Prototype high-speed optical delay line for stellar interferometry, Proc. SPIE, 1542, pp.205212, 1991.

3. M. Colavita et al., Potential of long-baseline infrared interferometry for narrow-angle astrometry, A \& A, 262, pp. 353-358, 1992.

4. A. Boden et al., An Interferometric Search for Bright Companions to 51 Pegasi, ApJL, 504, pp. L39-42, 1998. 